\title{
High Brightness Nano-patterned Photocathode Electron Sources for UEM
}

\author{
W.A. Schroeder,* J.A. Berger,* A.W. Nicholls, ${ }^{* *}$ N.J. Zaluzec, ${ }^{* * *}$ J. Hiller,*** and D.J. Miller***
}

* University of Illinois at Chicago, Department of Physics (m/c 273), 845 W. Taylor Street (rm. 2236), Chicago, IL 60607-7059

** Research Resources Center, University of Illinois at Chicago (m/c 337), 845 W. Taylor Street, Chicago, IL 60607

*** Electron Microscopy Center, Materials Science Division, Argonne National Laboratory, Argonne, IL 60439

Ultrafast electron microscopy (UEM) aims to combine the high spatial (sub-nm) resolution of electron microscopy with the high temporal (sub-ps) resolution afforded by today's ultrashort pulse lasers. Key to the development of such a research instrument is a laser-driven photoemission source with a high brightness; that is, a low normalized transverse emittance, $\varepsilon_{\mathrm{T}}=\Delta x . \Delta p_{x} /(m c)$, where $\Delta x$ and $\Delta p_{x}$ are the spatial size and transverse momentum width of the source respectively. However, $\Delta x$ is primarily determined by the required pulse charge for the experiment, which is limited to roughly $\varepsilon_{0} E(\Delta x)^{2}$, where $E$ is the photo-gun's electric field strength at the surface of the cathode. As a result, for a viable UEM, the value of $\Delta p_{x}$ will likely need to be reduced below that of conventional photoemission. A potential solution to this problem is to employ laser-driven plasmon-enhanced photoemission from a nano-patterned photocathode [1]; the idea being to use the strong oscillatory local surface plasmon field to 'control' the divergence of photoemitted electrons through the combined action of photoemission barrier suppression and ponderomotive acceleration [2].

Here, we present the observed properties of laser-driven plasmon-enhanced photoemission from a gold-coated, single-crystal silicon nano-patterned photocathode. Figure 1(a) shows the nanopatterned Si substrate with a $\sim 1 \mu \mathrm{m}$ period and a $\sim 10 \%$ (near sinusoidal) modulation depth produced by focused ion beam (FIB) milling using a defocused Ga ion beam in a Zeiss 1540XB FIB. Subsequent deposition of a $300 \mathrm{~nm}$-thick gold film provided the necessary nano-structure for the observation of resonant plasmon-enhanced photoemission at close to the expected $\theta \approx 41^{\circ}$ incidence angle (Figure 1(a) inset) evaluated for gold (dielectric constant, $\varepsilon=-3.95$ [3]) at $\lambda=523 \mathrm{~nm}-$ the green second harmonic of our high-power femtosecond $\mathrm{Yb}: \mathrm{KGW}$ laser [4] with a $2.37 \mathrm{eV}$ photon energy that is less than half the $\Phi=5.1 \mathrm{eV}$ work function for gold. The use of a substrate with a relatively high thermal conductivity to dissipate the heat generated by the laser-driven plasmon allowed for the continuous operation of the $63 \mathrm{MHz}$ pulsed electron source at an average incident laser intensity of $1 \mathrm{~kW} / \mathrm{cm}^{2}$. Prior efforts using a commercial gold grating (750 lines $\left./ \mathrm{mm}\right)$ as the nano-patterned photocathode under similar operational conditions were limited by the low thermal conductivity of the glass substrate (Figure 1(b)). The transverse momentum distribution of this laser-driven electron source is compared to that of a conventional Ta photocathode $(\Phi=4.25 \mathrm{eV})$ driven by the $261 \mathrm{~nm}(4.75 \mathrm{eV})$ fourth-harmonic of the $\mathrm{Yb}: \mathrm{KGW}$ laser using the scheme depicted in Figure 1(c), where the detector is located at the back focal plane (Fourier plane) of the magnetic lens system with focal length $f \approx 20 \mathrm{~cm}$.

References

[1] J. Kupersztych, et al., Phys. Rev. Lett. 86 (2001) 5180.

[2] J. Zawadzka, et al., Appl. Phys. Lett. 79 (2001) 2130. 
[3] P.B. Johnson and R.W. Christy, Phys. Rev. B 6 (1972) 4370.

[4] J.A. Berger, et al., Optics Express 16, 8629-8640.

[5] This work was supported by the National Science Foundation (DMR-0619573) and in part by the US DOE Office of Science, contract DE-AC02-06CH11357 at ANL. J.A. Berger acknowledges the support of a Department of Education GAANN Fellowship.

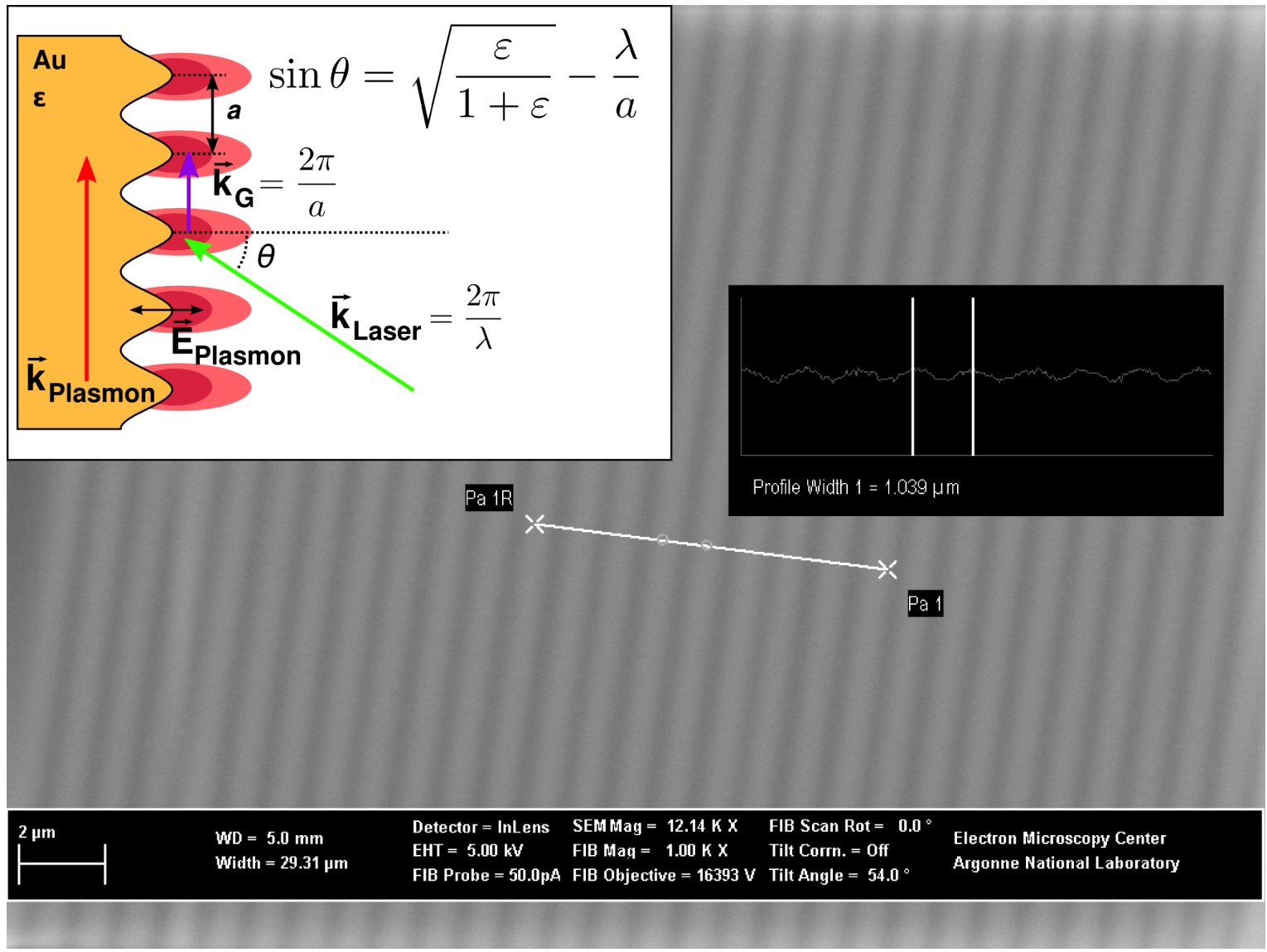

(a)

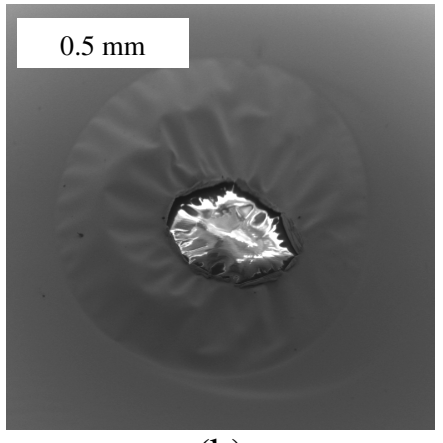

(b)

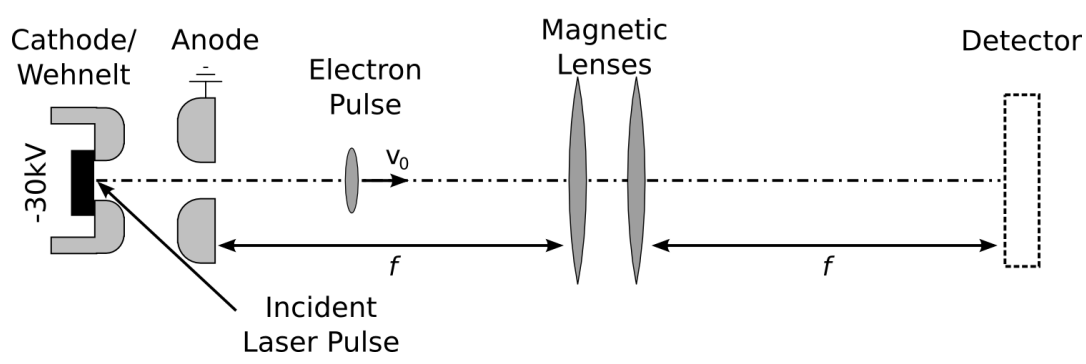

(c)

FIG 1. (a) High resolution SEM image of the FIB-milled sinusoidal nano-structure in single crystal Si. Inset: Laser-driven resonant surface plasmon excitation on a periodic gold surface. (b) SEM image of laser-induced damage (due to plasmon heating) on a commercial 750 lines $/ \mathrm{mm}$ gold grating. (c) Schematic of experiment designed to measure transverse momentum distribution of pulsed electron beam. 